\title{
A Murine Model for Disseminated Candidiasis in Neonates
}

\author{
NANCY Y. TSAI, SONIA S. LAFORCE-NESBITT, RICHARD TUCKER, AND JOSEPH M. BLISS
}

Department of Pediatrics [N.Y.T., S.S.L.-N., R.T., J.M.B.], Women \& Infants Hospital of Rhode Island, Warren Alpert Medical School of Brown University, Providence, Rhode Island 02905; Graduate Program in Pathobiology [J.M.B.], Brown University, Providence, Rhode Island 02912

\begin{abstract}
Candida albicans is the leading fungal pathogen causing invasive disease in immunocompromised patients including the neonate. A reliable animal model for disseminated candidiasis in the neonate is needed to study the unique aspects of this hostpathogen interaction. To establish such a model, 2-d-old BALB/c mouse pups were given i.p. injections with varied inocula of $C$. albicans or saline control. Pups were examined every 3-8 h for death. Surviving pups were killed at $72 \mathrm{~h}$. Kidney, lung, spleen, liver, and brain were homogenized and plated for colony counts and/or fixed for histological staining. The i.p. injection of $C$. albicans led to mortality in a dose-dependent fashion. Disseminated infection was confirmed by colony counts of homogenized kidney, lung, and brain, as well as by histological examination. Infection with a $C$. albicans mutant lacking the cell surface adhesin, Als3p, led to significant reduction in mortality relative to $\mathrm{WT}(p=0.03)$. This model will be useful to study the unique aspects of antifungal defense in a neonatal host and will provide a means to test novel therapeutic strategies. (Pediatr Res 69: 189-193, 2011)
\end{abstract}

$C$ andida albicans is the leading fungal pathogen in immunocompromised patients (1) and the third most common pathogen overall causing late-onset sepsis in premature infants (2). Well-described risk factors for disseminated disease in this population include gastrointestinal (GI) colonization, prolonged hospitalization, broad-spectrum antibiotic use, central venous catheters, and parenteral nutrition (3-5). Colonization of preterm infants has been documented to occur through both vertical (from mother to infant) and horizontal routes (6). Even with antifungal therapy, candidiasis is often fatal among premature infants and is associated with neurodevelopmental impairment among survivors. Follow-up examinations at $18-22$ mo corrected age show significant increases in rates of $\mathrm{CP}$, blindness, deafness, and mental retardation (7). The severity of these infections has led to development of prophylactic strategies to reduce colonization and limit invasive disease (8-11). Although effective, these strategies require prolonged exposure to antifungal agents with their associated risks $(12,13)$. Novel therapeutic strategies are needed to improve these outcomes. However, the mechanisms leading to immune compromise in the neonate are

Received July 28, 2010; accepted October 2, 2010

Correspondence: Joseph M. Bliss, M.D., Ph.D., Department of Pediatrics, Women \& Infants Hospital of Rhode Island, 101 Dudley Street, Providence, RI 02905; e-mail: jbliss@wihri.org

Supported by an NIH COBRE Grant P20 RR018728. likely different from other patient populations at risk (14). An animal model of disseminated candidiasis in a neonatal host is therefore needed to recapitulate unique aspects of this host-pathogen interaction.

Murine models have been a feasible and reliable method to study the pathogenesis of candidiasis but have their limitations. Unlike humans, the mouse is not naturally colonized in the GI tract with $C$. albicans. To achieve persistent colonization, the animals must be treated with antibiotics and/or immunosuppressive agents, and dissemination is uncommon $(15,16)$. Two strategies have been used to circumvent these issues. The most widely used model involves i.v. injection of adult animals via the lateral tail vein. This model has been used extensively to study virulence properties of the organism and immunological adaptations of the animal in response to hematogenous infection $(15,17)$. A second strategy is gastric inoculation of neonatal mice, which leads to persistent, albeit decreasing colonization over time with some dissemination and mortality. However, mortality was strain dependent and amounted to $\sim 50 \%$ or less in these studies $(18,19)$.

In this study, we sought to develop a model that would result in more reliable disease burden while still maintaining some clinical relevance. The goal was to avoid pharmacological immunosuppression so as to allow inquiry into inherent immune status in infected neonates and to obviate the variability and technical challenges inherent to GI colonization. GI pathology including abdominal surgery is an independent risk factor for disseminated disease $(3,5)$. When the integrity of the bowel mucosa is compromised, translocation of the organism is likely facilitated with spread via the enteric or lymphatic circulation. Direct inoculation of the peritoneum can also occur in the setting of bowel perforation with similar routes to dissemination. This study was structured to test the hypothesis that disseminated disease and subsequent mortality could be induced in a reliable and reproducible fashion by the i.p. route in neonatal mice without additional immunosuppression. This model provides the framework to study the unique host-pathogen interface in neonatal candidiasis and the development of novel therapeutic strategies.

Abbreviations: CFU, colony forming units; GI, gastrointestinal; scFv3, single-chain variable fragment 3 


\section{METHODS}

Strains and media. C. albicans strains used in this study include WT strain SC5314 (20) and strain 1843 containing a homozygous deletion in ALS3

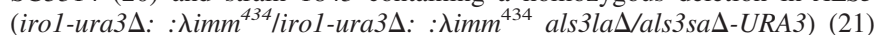
generously provided by Lois Hoyer. Starter cultures for injection were grown $16 \mathrm{~h}$ at $37^{\circ} \mathrm{C}$ with vigorous agitation in yeast extract peptone dextrose (YEPD) medium comprising $1 \%$ yeast extract, $2 \%$ peptone, and 2\% dextrose (Difco Laboratories, Becton, Dickinson and Company, Franklin Lakes, NJ). Cultures were predominantly (>99\%) yeast forms after this incubation. Before inoculation, overnight cultures of C. albicans were washed, enumerated on a hemacytometer, and resuspended in pyrogen-free saline (Hospira, Inc., Lake Forest, IL). The concentration was adjusted such that the desired unit dose per gram could be delivered in a volume of $10 \mu \mathrm{L}$.

Injection of neonatal mice. Timed pregnant $\mathrm{BALB} / \mathrm{c}$ mice were obtained from Charles River Laboratories (Wilmington, MA). Pregnant dams were maintained in individual cages with unlimited access to food and water. Mice were monitored to determine the date of parturition. Pups were delivered in litters ranging from 3 to 9 pups and were randomized before inoculation to either sterile saline (control) or $10^{\times}$colony forming units $(\mathrm{CFU}) / \mathrm{g}$ of $C$. albicans yeast. Randomization was performed within cages rather than by litter to account for maternal and litter variations. Although crosscontamination of pups assigned to different experimental groups was theoretically possible, this risk was minimized by the short duration of the experiment. Furthermore, in experiments using an endpoint such as mortality that is influenced by many variables, the risk of cross-contamination was outweighed by the risk of confounding effects of maternal and litter variability inherent to a litter-based randomization scheme. Pups were injected on postpartum d 2. Just before injection, each pup was weighed to the nearest 10th of a gram. Weight of pups was closely clustered around $2 \mathrm{~g}$, so each pup received a standard dose of $20 \mu \mathrm{L}$ yeast or sterile saline injected i.p. in the lower half of the abdomen. After injection, pups were examined every $3-8 \mathrm{~h}$ for death or signs of illness. The pups were dissected at the time of natural death or killed for dissection when found moribund. All surviving animals were killed at $72 \mathrm{~h}$ after injection. A single kidney and lung were harvested and immediately homogenized for colony counts. If the time of natural death could not be accurately determined within $2 \mathrm{~h}$, the organ colony counts were excluded from analysis. The remaining kidney, lung, spleen, liver, and brain were fixed in $10 \%$ buffered formalin (Fisher Scientific, Kalamazoo, MI) for subsequent histology. In selected studies, brain tissue was also collected for homogenization and colony counts.

Selected tissues underwent histological preparation and silver staining at the institutional core facility. Kidney, lung, and brain were homogenized with a FastPrep-24 Instrument (MP Biomedicals, Inc., Solon, OH) using Lysing Matrix D (Qbiogene; MP Biomedicals, Inc.) in 1-mL sterile saline, and appropriate dilutions were plated on YEPD. Colony counts were performed after an overnight incubation at $37^{\circ} \mathrm{C}$. All animal studies were reviewed and approved by the Lifespan Institutional Animal Care and Use Committee, which oversees the animal care facility where animals were housed for this study.

\section{RESULTS}

C. albicans infection in neonatal mice. Mouse pups were injected i.p. on postpartum d 2 with concentrations of $C$. albicans strain SC5314 ranging from $10^{4}$ to $10^{8} \mathrm{CFU} / \mathrm{g}$ or with saline. Mice were followed closely for signs of illness and killed at $72 \mathrm{~h}$ after injection. Figure 1 shows the Kaplan-Meier survival curve summarizing these experiments. Doses of $10^{6}$ $\mathrm{CFU} / \mathrm{g}$ and below were not associated with mortality and caused no apparent clinical symptoms. Doses above $10^{6}$ $\mathrm{CFU} / \mathrm{g}$ caused increased mortality in a dose-dependent fashion. Because $10^{7} \mathrm{CFU} / \mathrm{g}$ led to near complete mortality by study end point with a range of time of death throughout the observation period, this dose was selected for subsequent experiments.

Tissue sections of kidney, spleen, liver, and brain were silver stained to detect fungal elements (Fig. 2). Yeast and hyphal elements were scattered in the kidney and liver parenchyma with no specific anatomic relationship (Fig. $2 A$ and $B$ ). There was no fungus detected in brain by histology (not shown). In the low

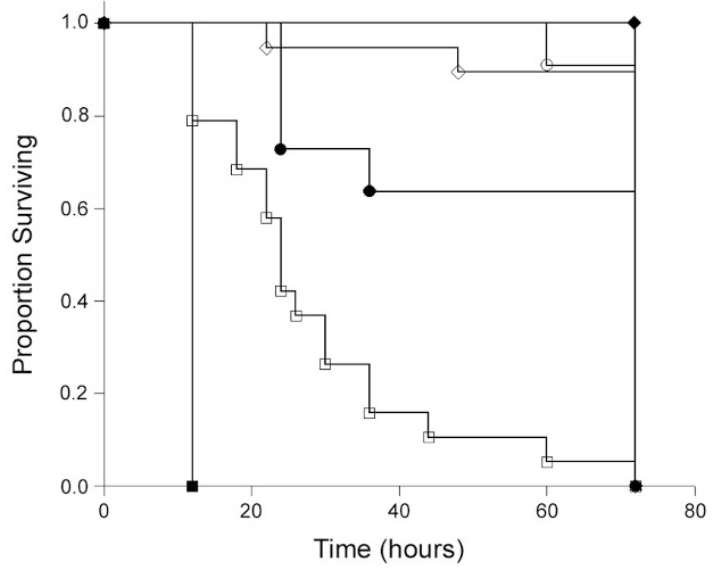

Figure 1. Kaplan-Meier survival curve by dose injected. Two-day-old mouse pups were injected i.p. with the following doses of WT C. albicans, and survival curves were plotted: $\mathbf{\square}, 1 \times 10^{8} \mathrm{CFU} / \mathrm{g}(n=3) ; \square, 1 \times 10^{7}$ $\mathrm{CFU} / \mathrm{g}(n=19) ; \bullet, 5 \times 10^{6} \mathrm{CFU} / \mathrm{g}(n=11) ; \bigcirc, 1 \times 10^{6} \mathrm{CFU} / \mathrm{g}(n=11) ;$ $\diamond, 1 \times 10^{4} \mathrm{CFU} / \mathrm{g}(n=3)$; and $\diamond$, saline $(n=19)$. Mortality occurred in a dose-dependent fashion at doses higher than $10^{6} \mathrm{CFU} / \mathrm{g}$.

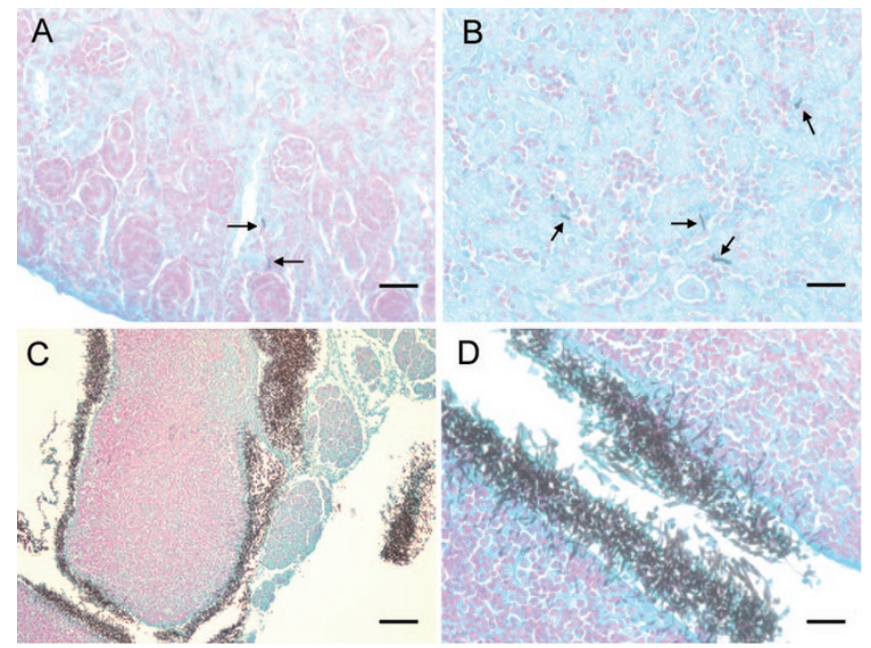

Figure 2. Tissue histology. Silver stain of representative sections from kidney $(A)$, liver $(B)$, and spleen $(C, \times 10$ magnification and $D, \times 40$ magnification) are depicted from an animal injected with $10^{8} \mathrm{CFU} / \mathrm{g}$ WT $C$. albicans. Arrows indicate hyphal elements visible within the organ parenchyma. Heavy involvement of the capsular and subcapsular regions of the spleen was seen. $A, B$, and $D:$ bar $=25 \mu \mathrm{m} ; C:$ bar $=100 \mu \mathrm{m}$.

power view of the spleen, abundant fungal elements were diffusely present in the capsular region (Fig. 2C). A high power view of the same region demonstrated prominent hyphae with penetration into the spleen parenchyma (Fig. 2D).

Colony counts were obtained from homogenized kidney and lung and were highly variable (Table 1). In general, colony counts were higher in kidney compared with lung at a given dose, and extent of fungal burden was proportional to dose injected. Statistical analysis using a negative binomial model supported this dose-response relationship with $p=$ 0.0005 for kidney and $p=0.003$ for lung. Although no fungal elements were detected in histological studies of the brain, an additional experiment was conducted to examine brain involvement by colony counts, a more sensitive measure. Among 12 pups injected, all died by study end point, and nine 
Table 1. Tissue burden by dose of WT Candida albicans injected

\begin{tabular}{|c|c|c|c|c|c|c|c|c|c|}
\hline \multirow[b]{2}{*}{ Dose (CFU/g) } & \multicolumn{4}{|c|}{ Kidney (CFU/Organ) } & \multicolumn{4}{|c|}{ Lung (CFU/Organ) } & \multirow[b]{2}{*}{$n$} \\
\hline & Mean & Median & Minimum & Maximum & Mean & Median & Minimum & Maximum & \\
\hline $10^{7}$ & 10,442 & 600 & 0 & 71,300 & 1446 & 500 & 0 & 6100 & 13 \\
\hline $5 \times 10^{6}$ & 8734 & 70 & 0 & 66,000 & 1055 & 30 & 0 & 7800 & 8 \\
\hline $10^{6}$ & 806 & 20 & 0 & 8000 & 20 & 25 & 0 & 40 & 11 \\
\hline $10^{4}$ & 13 & 20 & 0 & 20 & 0 & 0 & 0 & 0 & 3 \\
\hline
\end{tabular}

pups $(75 \%)$ had colony counts ranging from 100 to 240 colonies per brain. Although a lower fungal burden was seen relative to kidney and lung, these data support some involvement of the CNS. No organisms were recovered from any organs taken from animals in the saline control groups, providing reassurance in regard to the possibility of crosscontamination among animals in the same litter.

Assessment of ALS3 mutant in neonatal model. To determine the utility of this model in assessing virulence determinants of $C$. albicans, a mutant (1843) carrying a homozygous deletion of the adhesin gene, $A L S 3$, was evaluated in the neonatal mouse model. Previous work demonstrated that this strain had reduced adhesion to epithelial and endothelial cells in vitro (22). The als 3 mutant strain yielded a statistically significant reduction in mortality relative to WT (Fig. 3, $p=$ 0.03 ). The median survival for the WT was $24 \mathrm{~h}$ compared with $44 \mathrm{~h}$ for the mutant. Tissue burden in kidney and lung was also compared in these animals (Table 2). Because colony count data were again highly disperse and not normally distributed, a negative binomial model was used for analysis. Once again, tissue burden was higher in kidney than in lung for both strains. Although trends toward higher colony counts in mice injected with WT versus mutant could be identified, there was no significant difference in tissue fungal burden in these animals.

\section{DISCUSSION}

Invasive candidiasis portends a poor prognosis despite available antifungal agents. A sophisticated understanding of

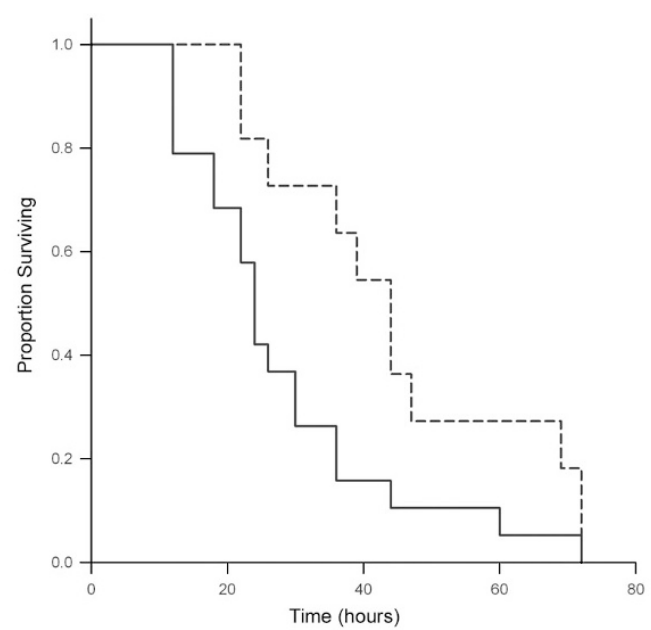

Figure 3. Kaplan-Meier survival curve comparing WT C. albicans and als3 deletion mutant. Two-day-old mouse pups were injected i.p. with $10^{7} \mathrm{CFU} / \mathrm{g}$ WT C. albicans (solid line, $n=19$ ) or als3 deletion mutant (dashed line, $n=$ 11 ), and survival curves were plotted. Median survival was $24 \mathrm{~h}$ and $44 \mathrm{~h}$, respectively ( $p=0.03$ by log-rank test). host-pathogen interactions will be required to make additional progress in treatment and prevention of these infections. The model described here will be useful in studies to explore the unique aspects of the neonatal host in this disease. Neonatal mouse models have been successfully used to study sepsis with other microorganisms including group B Streptococcus (GBS) (23), Escherichia coli (24), Pseudomonas aeruginosa (25), and Listeria monocytogenes (26,27). In some cases, these studies have uncovered significant differences that can be defined between the neonatal and adult host and demonstrate the importance of neonatal models to study invasive disease.

Previous studies of $C$. albicans in the neonatal mouse used gastric inoculation as the route of infection. By using neonatal mice, Pope et al. (18) provided the first report of lethal candidiasis in an animal model after GI colonization without any additional measures to compromise immunity in the animals. In this study, 5- to 6-d-old mouse pups were inoculated via intragastric injection $\left(5 \times 10^{8} \mathrm{CFU}\right)$, and systemic spread of infection was seen in selected organs. Fungal invasion was seen in liver, kidney, and spleen within $6 \mathrm{~h}$ of inoculation, suggesting timely passage across the digestive tract wall or entry into the systemic circulation, possibly through the lymphatics. However, mortality was $\sim 50 \%$ or less in this model and tissue burden decreased over time. A subsequent study using a lower dose $\left(2 \times 10^{7} \mathrm{CFU}\right)$ in 6-d-old pups by the same route also led to recovery of $C$. albicans from kidney, liver, lung, and spleen but in relatively smaller numbers and no mortality (19). A dose of $1 \times 10^{7} \mathrm{CFU}$ led to still fewer or no recovery of fungi from these organs. However, long-term GI colonization was demonstrated in these animals and early colonization led to protective immune responses after later IV challenge with $C$. albicans as adults. In another study, 6-d-old mouse pups were orally inoculated with $C$. albicans after cortisone-induced immunosuppression. Histology of the entire GI tract showed highest frequency of invasion of mucosa by Candida in the jejunum. Only GI tract organs were studied in this model (28).

GI colonization models using adult mice show important differences from the neonatal models. Broad-spectrum antibiotic administration for $3 \mathrm{~d}$ before oral inoculation with $C$. albicans was necessary for GI colonization to develop (29). In addition, once colonized, extraintestinal dissemination in these animals was infrequent. However, when these animals were treated with dexamethasone, dissemination to kidney and mesenteric lymph nodes did occur but mortality remained low (16). Features of disease associated with infection by the i.p. route in adult mice are also available. Vonk et al. (30) studied the role of TNF- $\alpha$ and lymphotoxin (LT)- $\alpha$ by injecting $C$. 
Table 2. Tissue burden in mice infected with WT vs mutant (als3-/-) C. albicans

\begin{tabular}{|c|c|c|c|c|c|c|c|c|}
\hline & \multicolumn{4}{|c|}{ WT (CFU/organ), $n=13$} & \multicolumn{4}{|c|}{ als $3-/-(\mathrm{CFU} /$ organ $), n=10$} \\
\hline & Mean & Median & Minimum & Maximum & Mean & Median & Minimum & Maximum \\
\hline Kidney* & 10,442 & 600 & 0 & 71,300 & 3950 & 1100 & 0 & 13,500 \\
\hline Lung $\dagger$ & 1446 & 500 & 0 & 6100 & 518 & 350 & 0 & 2200 \\
\hline
\end{tabular}

$* p=0.29$ for WT $v s$ mutant.

$\dagger p=0.14$ for WT $v s$ mutant.

albicans i.p. in adult TNF-LT double knockout mice and their WT littermates. Unlike this study in neonates, disseminated disease only occurred if immunosuppression was induced with cyclosporine before infection. Otherwise, the adult mice formed local abscesses that were cleared without disseminated disease. Taken together, these studies demonstrate that features of candidiasis in mouse models differ dramatically in the neonate compared with the adult, likely because of the relative immaturity of host defenses in the neonatal period. Such differences support the notion that features of disease unique to the neonatal host can be manifested by such an approach. However, there are additional factors placing preterm infants at risk for disseminated candidiasis that will be difficult to emulate in a murine model. Interventions such as indwelling catheters, parenteral nutrition, lack of enteral nutrition/breast milk, and many others that increase risk in the NICU are difficult to model and somewhat limit the applicability of host defense studies at this stage of mouse development to that of the preterm human.

In this study, the heavy involvement of the spleen with scattered foci in other organs supports a hematogenous route of dissemination, perhaps initiating in the spleen. Although involvement of the spleen was detected in the neonatal gastric inoculation model, colonization was similar or less than other organs (18). Presumably, the i.p. route of infection is responsible for the heavy spleen involvement in our model, either by direct contact with the organ or through lymphatic channels. Brieland et al. (31) inoculated C. albicans $\left(5 \times 10^{6} \mathrm{CFU} /\right.$ mouse) via lateral tail vein injections into adult, immunocompetent mice and reported growth of $C$. albicans in various organs. The kidney was noted to have logarithmic growth in fungal burden. However, the liver and heart fungal burden declined quickly over time. The brain, lung, and spleen were all noted to have steady fungal loads with no significant change over the duration of the infection. Consistent with these data, the kidney counts in this study were higher than in lung tissue. The study by Brieland et al. collected data over a 21-d postinfection time course. In our model, mortality occurred within $72 \mathrm{~h}$, and any surviving pups had generally cleared the infection by the 72-h time point. The kinetics of infection were therefore quite different from the model of Brieland et al.

The study by Brieland et al. described multiple foci of hyphal invasion in kidneys, hearts, brains, and spleens of infected mice, with the largest fungal burden in the kidney. We found the largest foci of hyphae around the splenic capsule. We also did not visualize hyphal elements in brain of neonatal mice by histology, whereas the adult model showed brain involvement within $48 \mathrm{~h}$ postinfection. Brain colony counts yielded consistent but reduced fungal burdens when compared with lung and kidney, suggesting that the fungal burden of the brain is not high enough to be detected by histology. Because tissue homogenates were the only way to assess fungal burden, involvement of vascular structures in the brain rather than the parenchyma itself is also possible. The differences in tissue distribution between these models likely relate to the route of infection and/or the dose of inoculation but may also be influenced by developmental stage of the animal.

We have previously described a single-chain variable fragment 3 (scFv3), which is specific to Als3p (22). Als3p is a cell wall protein expressed on $C$. albicans hyphae, which belongs to the Als family of adhesins (32). Als3p enables adherence to both epithelial and endothelial host cells through interaction with E-cadherin and N-cadherin, respectively, (33). Strain 1843 , carrying a homozygous deletion of $A L S 3$, demonstrates reduced adhesion to human epithelial and endothelial cells. Treatment of WT C. albicans with scFv3 resulted in reduced adherence, similar to the als3 mutant (22). In our model, the als3 deletion mutant showed somewhat attenuated mortality. Antibodies against Als3p such as scFv3 may therefore be useful to confer protection from disseminated candidiasis. This model provides fertile grounds to test this and other therapeutic strategies. Experiments are underway to evaluate $\mathrm{scFv} 3$ and other Als3p-specific antibodies for their capacity to provide protection. Novel chemotherapeutic agents against fungi could also be tested in this model for efficacy and to assess any unique toxicity that may arise in a neonatal setting. Studies of pathogenesis with $C$. albicans frequently find differences among strains. This model can be used to extend these observations and make comparisons among isolates that are presumed to be different in pathogenic potential. In addition, as non-albicans species increase in prevalence in the NICU, this model will have utility to compare the pathogenic features of the different Candida species and potentially tailor appropriate therapies.

Acknowledgments. We thank Lois Hoyer for providing strain 1843 and helpful discussions and Monique DePaepe for pathological interpretation of histology.

\section{REFERENCES}

1. Pfaller MA, Diekema DJ 2007 Epidemiology of invasive candidiasis: a persistent public health problem. Clin Microbiol Rev 20:133-163

2. Stoll BJ, Hansen N, Fanaroff AA, Wright LL, Carlo WA, Ehrenkranz RA, Lemons JA, Donovan EF, Stark AR, Tyson JE, Oh W, Bauer CR, Korones SB, Shankaran S, Laptook AR, Stevenson DK, Papile LA, Poole WK 2002 Late-onset sepsis in very low birth weight neonates: the experience of the NICHD Neonatal Research Network. Pediatrics 110:285-291 
3. Saiman L, Ludington E, Dawson JD, Patterson JE, Rangel-Frausto S, Wiblin RT, Blumberg HM, Pfaller M, Rinaldi M, Edwards JE, Wenzel RP, Jarvis W 2001 Risk factors for Candida species colonization of neonatal intensive care unit patients. Pediatr Infect Dis J 20:1119-1124

4. Reef SE, Lasker BA, Butcher DS, McNeil MM, Pruitt R, Keyserling H, Jarvis WR 1998 Nonperinatal nosocomial transmission of Candida albicans in a neonatal intensive care unit: prospective study. J Clin Microbiol 36:1255-1259

5. Feja KN, Wu F, Roberts K, Loughrey M, Nesin M, Larson E, Della-Latta P, Haas J, Cimiotti J, Saiman L 2005 Risk factors for candidemia in critically ill infants: a matched case-control study. J Pediatr 147:156-161

6. Bliss JM, Basavegowda KP, Watson WJ, Sheikh AU, Ryan RM 2008 Vertical and horizontal transmission of Candida albicans in very low birth weight infants using DNA fingerprinting techniques. Pediatr Infect Dis J 27:231-235

7. Benjamin DK Jr, Stoll BJ, Fanaroff AA, McDonald SA, Oh W, Higgins RD, Duara S, Poole K, Laptook A, Goldberg R 2006 Neonatal candidiasis among extremely low birth weight infants: risk factors, mortality rates, and neurodevelopmental outcomes at 18 to 22 months. Pediatrics 117:84-92

8. Kaufman D, Boyle R, Hazen KC, Patrie JT, Robinson M, Donowitz LG 2001 Fluconazole prophylaxis against fungal colonization and infection in preterm infants. N Engl J Med 345:1660-1666

9. Manzoni P, Stolfi I, Pugni L, Decembrino L, Magnani C, Vetrano G, Tridapalli E, Corona G, Giovannozzi C, Farina D, Arisio R, Merletti F, Maule M, Mosca F, Pedicino R, Stronati M, Mostert M, Gomirato G 2007 A multicenter, randomized trial of prophylactic fluconazole in preterm neonates. N Engl J Med 356:2483-2495

10. Kaufman D, Boyle R, Hazen KC, Patrie JT, Robinson M, Grossman LB 2005 Twice weekly fluconazole prophylaxis for prevention of invasive Candida infection in high-risk infants of $<1000$ grams birth weight. J Pediatr 147:172-179

11. Clerihew L, Austin N, McGuire W 2007 Prophylactic systemic antifungal agents to prevent mortality and morbidity in very low birth weight infants. Cochrane Database Syst Rev 4:CD003850

12. Long SS, Stevenson DK 2005 Reducing Candida infections during neonatal intensive care: management choices, infection control, and fluconazole prophylaxis. J Pediatr 147:135-141

13. Manzoni P, Mostert M, Jacqz-Aigrain E, Farina D 2009 The use of fluconazole in neonatal intensive care units. Arch Dis Child 94:983-987

14. Levy O 2007 Innate immunity of the newborn: basic mechanisms and clinical correlates. Nat Rev Immunol 7:379-390

15. Clancy CJ, Cheng S, Nguyen MH 2009 Animal models of candidiasis. Methods Mol Biol 499:65-76

16. Bendel CM, Wiesner SM, Garni RM, Cebelinski E, Wells CL 2002 Cecal colonization and systemic spread of Candida albicans in mice treated with antibiotics and dexamethasone. Pediatr Res 51:290-295

17. Romani L 2002 Immunology of invasive candidiasis. In: Calderone RA (ed) Candida and Candidiasis. ASM Press, Washington, DC, pp 223-241

18. Pope LM, Cole GT, Guentzel MN, Berry LJ 1979 Systemic and gastrointestinal candidiasis of infant mice after intragastric challenge. Infect Immun 25:702-707
19. Domer JE 1988 Intragastric colonization of infant mice with Candida albicans induces systemic immunity demonstrable upon challenge as adults. J Infect Dis 157:950-958

20. Gillum AM, Tsay EY, Kirsch DR 1984 Isolation of the Candida albicans gene for orotidine-5' -phosphate decarboxylase by complementation of $S$. cerevisiae ura3 and E. coli pyrF mutations. Mol Gen Genet 198:179-182

21. Zhao X, Oh SH, Cheng G, Green CB, Nuessen JA, Yeater K, Leng RP, Brown AJ, Hoyer LL 2004 ALS3 and ALS8 represent a single locus that encodes a Candida albicans adhesin; functional comparisons between Als3p and Als1p. Microbiology 150:2415-2428

22. Laforce-Nesbitt SS, Sullivan MA, Hoyer LL, Bliss JM 2008 Inhibition of Candida albicans adhesion by recombinant human antibody single-chain variable fragment specific for Als3p. FEMS Immunol Med Microbiol 54:195-202

23. Rodewald AK, Onderdonk AB, Warren HB, Kasper DL 1992 Neonatal mouse model of group B streptococcal infection. J Infect Dis 166:635-639

24. Cox F, Taylor L 1990 Prevention of Escherichia coli K1 bacteremia in newborn mice by using topical vaginal carbohydrates. J Infect Dis 162:978-981

25. Tang HB, DiMango E, Bryan R, Gambello M, Iglewski BH, Goldberg JB, Prince A 1996 Contribution of specific Pseudomonas aeruginosa virulence factors to pathogenesis of pneumonia in a neonatal mouse model of infection. Infect Immun 64:37-43

26. Chen Y, Nakane A, Minagawa T 1989 Recombinant murine gamma interferon induces enhanced resistance to Listeria monocytogenes infection in neonatal mice. Infect Immun 57:2345-2349

27. Genovese F, Mancuso G, Cuzzola M, Biondo C, Beninati C, Delfino D, Teti G 1999 Role of IL-10 in a neonatal mouse listeriosis model. J Immunol 163:2777-2782

28. Cole GT, Seshan KR, Pope LM, Yancey RJ 1988 Morphological aspects of gastrointestinal tract invasion by Candida albicans in the infant mouse. J Med Vet Mycol 26:173-185

29. Kinneberg KM, Bendel CM, Jechorek RP, Cebelinski EA, Gale CA, Berman JG, Erlandsen SL, Hostetter MK, Wells CL 1999 Effect of INT1 gene on Candida albicans murine intestinal colonization. J Surg Res 87:245-251

30. Vonk AG, Netea MG, van Krieken JH, van der Meer JW, Kullberg BJ 2002 Delayed clearance of intraabdominal abscesses caused by Candida albicans in tumor necrosis factor-alpha- and lymphotoxin-alpha-deficient mice. J Infect Dis 186:1815-1822

31. Brieland J, Essig D, Jackson C, Frank D, Loebenberg D, Menzel F, Arnold B, DiDomenico B, Hare R 2001 Comparison of pathogenesis and host immune responses to Candida glabrata and Candida albicans in systemically infected immunocompetent mice. Infect Immun 69:5046-5055

32. Hoyer LL 2001 The ALS gene family of Candida albicans. Trends Microbiol 9:176-180

33. Phan QT, Myers CL, Fu Y, Sheppard DC, Yeaman MR, Welch WH, Ibrahim AS, Edwards JE Jr, Filler SG 2007 Als3 is a Candida albicans invasin that binds to cadherins and induces endocytosis by host cells. PLoS Biol 5:e64 